127 
(Aus der Arbeitsstätte für Züchtungsforschung, Luckenwalde.)

\section{Zielsetzung und Probleme in der Speisemöhrenzüchtung ${ }^{1}$. Von Gerda Krawitter und R. v. Sengbusch.}

\section{Zuchtziele.}

I. Zuchtziele, die der Anbauer stellt. Die Möhre ist eine unserer wichtigsten Wurzelgemüsearten. Im Feld- und Erwerbsgartenbau wurden I939 rund Io ooo ha Möhren angebaut. Wie groß der Anbau der Möhren im Kleingarten ist, läßt sich nicht mit Sicherheit feststellen. Die Fläche dürfte aber mindestens 20000 ha betragen. Bei einer Mindestgesamtfläche von etwa 30000 ha Möhren beläuft sich die erforderliche Saatgutmenge auf etwa I000 bis $1500 \mathrm{dz}$.

Über die Art des Anbaues der Möhre ist folgendes zu sagen:

a) Der Anbau von Treibmöhren. Die Möhren werden frühzeitig in warmen Kästen oder auch im Gewächshaus ausgesät und liefern so die erste Möhrenfrischernte des Jahres. Für diese Art des Anbaues werden Treibmöhrensorten verwendet.

b) Der Anbau von frühreifen Freilandmöhren. Den Anschluß an die sehr frühen Treibmöhren

\footnotetext{
1 Die Arbeiten wurden mit Unterstützung des Forschungsdienstes durchgeführt.
}

bilden die frühen Freilandmöhren. Diese werden möglichst frühzeitig ins Freiland gesät. Für den Anbau frühreifer Freilandmöhren werden entsprechende Frühsorten verwendet. Diese Sorten eignen sich auch für die Spätaussaat.

c) Der Anbau von späten, sehr ertragreichen, lagerfähigen Möhren. Die frühgesäten Freilandmöhren werden, wenn man sie bis zum Herbst im Boden läßt, überständig. Man baut daher für die Ernte im Herbst sehr ertragreiche, spätreifende Möhren an. Diese werden nicht nur unmittelbar nach der Ernte im Herbst genutzt, sondern auch eingelagert. Sie ermöglichen dann einen Frischverbrauch im Laufe des Winters und Frürijahrs.

Für den Anbau im Kleingarten kommen eigentlich nur die beiden letztgenannten Gruppen, die ,frühreife Freilandmöhre" und die ,,spätreifende, ertragreichc und gleichzeitig lagerfähige Freilandmöhre" in Frage.

Der Anbauer hat bei den Treib- und Frühmöhren ein Interesse an möglichst frühzeitigen, hohen Erträgen, bei den Spätmöhren allein am 
Massenertrag. Ferner möchte der Anbauer eine Möhre haben, die nicht zu feines Laub entwickelt, weil bei diesen die Entwicklung zögernder ist, und das Sauberhalten der Äcker größere Mühe macht als bei groblaubigen Möhren. Die Möhren sollen im ersten Jahr nicht schossen, damit Ausfälle im Ertrag vermieden werden. Eine weitere unangenehme Eigenschaft der Möhren, die erhebliche Ernteverluste verursacht, ist dasPlatzen der Möhren. Auch bei feuchter Witterung sollen die Möhren nicht platzen. Die späten Möhren sollen lagerfähig sein, damit Verluste im Winterlager nicht eintreten.

2. Zuchtziele, die der Konservenfabrikant stellt. Die Möhre ist wegen ihrer verhältnismäßig guten Lagerfähigkeit eigentlich keine typische Kulturart der Konservenindustrie. Trotzdem werden die Möhren in erheblichem Umfang in der Naß- und Trockenkonservenindustrie verarbeitet. Die Gefrierindustrie lehnt heute die Konservierung der Möhre ab, aber es ist durchaus möglich, daß in Zukunft auch von der Gefrierindustrie feine Qualitäten von Möhren eingefroren werden. Die Naßkonservenindustrie konserviert die Möhren in sehr erheblichem Umfang zusammen mit Erbsen, daneben aber auch in reiner Form. Heute sind für die Herstellung von Erbsen/Möhren-Mischgemüse die kugelförmigen Möhren (Sorte „Pariser Markt") besonders geschätzt. Für die Massenkonservierung von Möhren kommen sowohl bei der Naßkonserven- als auch bei der Präservenindustrie die späten ertragreichen Möhren in Frage.

Nach unseren heutigen Feststellungen können wir für eine Konservenmöhre nachfolgende Zuchtziele aufstellen:

a) Außerlich sichtbare Eigenschaften. Spitzköpfig, damit die Reinigung der Möhren leicht vonstatten gehen kann, im Gegensatz zu senkköpfig. Zylindrisch im Gegensatz zu keilförmig, damit der Abfall gering ist. Keilförmige Möhren ergeben beim Schneiden einen höheren Abfallprozentsatz. Außerdem weisen keilförmige Möhren leichter einen hohlen Holzteil auf. Die äußere Form soll glatt im Gegensatz zu gefurcht sein. Die Wurzelansätze sollen möglichst klein und fein sein im Gegensatz zu groben vertieften Wurzelansätzen. Die Seitenwurzeln selbst sollen so fein sein, daß die Möhre nach der Ernte nicht ,,bärtig" erscheint. Ferner sollen die Möhren nicht grünköpfig und nicht grün in der kambialen Zone zwischen Holz- und Rindenteil sein und nicht hoch aus dem Boden wachsend, damit die Gefahr des Grünköpfigwerdens nach Möglichkeit ausgeschaltet wird.

b) Eigenschaften des Rübenkörpers. Der Holzteil Der Züchter, I5. Jahrg.
(Herz) soll nach Möglichkeit klein sein und in seiner Struktur dem des Rindenteils gleichen. Der Holzteil soll möglichst feine Ausläufer durch den Rindenteil treiben, so daß der Rindenteil nicht mit dicken Strängen durchsetzt erscheint. Die kambiale Zoine zwischen Holz-und Rindenteil soll möglichst dünn sein. Der Holzteil soll fest mit der Rinde verbunden sein und sich nicht von ihm lösen. Der Holzteil soll auch bei Überreife nicht hohl werden, weil er sonst zu inneren Zerstörungen Anla $\beta$ geben kann.

Bezüglich der Farbe wünschen wir, daß der Holzteil und die kambiale Zone die gleiche rote Farbe wie der Rindenteil aufweisen, daß die Farbe möglichst tief dunkelrot ist, und $\mathrm{da} B$ diese tief dunkelrote Farbe bei der Konservierung und im Laufe der Lagerung nicht verblaßt oder besonders bei der Konservierung nicht in gelb umschlägt.

Zum Schluß hat der Konservenfabrikant, da er auch Möhren einlagert, genau wie der Landwirt ein Interesse daran, daß die Möhren gut lagerfähig sind.

Der Naßkonservenfabrikant ist in der Lage, den Geschmack der Möhren durch Zusatz von Zucker zu verbessern. Diese Möglichkeit besteht bei der Herstellung von Trocken- und Gefriergemüse nicht. Man wird daher insbesondere für die Trocken- und Gefrierindustrie Möhrensorten züchten müssen, die von Haus aus einen guten Geschmack, insbesondere aber einen hohen Sü $\beta$ wert haben. Ferner wird in der Trocken- und Gefrierindustrie die Frage der positiven und negativen Geschmacksstoffe eine große Rolle spielen. Auch diese bleiben in der Rohware nach der Verarbeitung erhalten und können sich in gutem und schlechtem Sinn bei der Speiseherstellung auswirken. Es müssen daher Sorten gezüchtet werden, die reich an positiven und arm an negativen Geschmacksstoffen sind.

3. Zuchtziele, die der Verbraucher stellt. Von unseren Gemüsearten nimmt die Möhre bezüglich des Karotingehalts (Provitamin A) eine Sonderstellung ein. Sie hat einen sehr hohen Karotingehalt und liefert außerdem hohe Flächenerträge an Karotin. Die Gesundheitsführung ist daher gerade an dieser Eigenschaft der Möhre besonders interessiert, und sie wird daher als Zuchtziel die Steigerung des Karotinertrags je Flächeneinheit aufstellen. Dieses Zuchtziel ist für die einzelnen Möhrentypen „frühe Treibmöhren", „frühe Freilandmöhren", ,,späte und lagerfähige Freilandmöhren" entsprechend zu modifizieren. Die Früh- und Spätmöhren sollen zurZeit ihrer wirtschaftlichenReife die Höchsterträge an Karotin liefern. Die lagerfähigen Möhren sollen aber nicht nur im Herbst 
Höchstvitaminerträge besitzen, sondern sie sollen außerdem einen möglichst geringen Karotinverlust über Winter aufweisen.

Finnische Arbeiten (VIRTANEN) zeigen, daß von dem chemisch nachweisbaren Karotin nicht alles vom Darm resorbiert wird. Bei den Möhren liegen diese Verhältnisse besonders ungünstig. VIRTANEN stellt fest, daß beim Verzehr von frischen oder gekochten Möhren nur 5\% des Karotins resorbiert werden, und daß dieser Prozentsatz steigt, wenn man die Möhren ganz fein zerkleinert, aber auch dann erhöht sich der Prozentsatz nur auf etwa $20 \%$.

Es ist Tatsache, daß von dem in der Möhre vorhandenen Karotin nur ein kleiner Teil vom Körper aufgenommen wird. Züchterisch ergeben sich daraus große Schwierigkeiten. Es nützt praktisch nichts, den chemisch nachweisbaren Karotingehalt bzw. -ertrag zu erhöhen, sondern man muß den biologisch verwertbaren Karotingehalt bzw. -ertrag steigern. Es wird daher nicht genügen, chemische Methoden in der Züchtung anzuwenden. In Vorversuchen wird die Frage $z u$ klären sein, ob eine Beziehung zwischen dem gesamten chemisch nachweisbaren Karotingehalt und dem biologisch verwertbaren Teil des Karotingehalts besteht. Wenn eine Korrelation zwischen diesen beiden Größen bestehen sollte, so wird man sich evtl. vorläufig mit der chemischen Methode der Karotinbestimmung begnügen können.

Die Gesundheitsführung wird demnach gerade diese Verhältnisse besonders berücksichtigen und als endgültiges Zuchtziel bei den Möhren bezüglich des Karotingehalts und -ertrags einen möglichst hohen biologisch verwertbaren Karotinertrag je Flächeneinheit aufstellen.

Ferner legt der Verbraucher Wert auf einen guten Geschmack. Dieser setzt sich zusammen aus hohem Zuckergehalt, hohem Gehalt an angenehmen Aromastoffen, Abwesenheit von unangenehmen Geschmacksstoffen. Wichtig ist ferner, daß der gute Geschmack bei den späten Möhren auch nach längerer Lagerzeit erhalten bleibt. Zum Geschmack gehören aber nicht nur der Geschmacksstoffgehalt, sondern auch die morphologische Struktur des Gewebes. Die Möhre darf weder holzig noch zu strukturlos sein, um gut zu schmecken. Eine spezifische Möhrenkonsistenz wird angestrebt.

\section{Methoden.}

I. Methoden zur Erfassung der Eigenschaften, die den Anbauer interessieren.

Ertrag. Die Technik der Ertragsprüfung bei Möhren ist heute noch nicht so weit entwickelt, $\mathrm{da} B$ wir eindeutige Angaben über die Versuchs- anstellung machen können. Auf Grund der bisherigen Erfahrungen können wir folgende Richtlinien aufstellen: Zunächst hat der Züchter die Ertragsleistung von Einzelpflanzen- bzw. Paarnachkommenschaften zu prüfen. :Eine Einzelpflanze liefert nur wenige Gramm Saatgut. Dieses reicht aus, um I oder 2 zweireihige Parzellen von 3-4 m Länge auszulegen. Der Möhrenzüchter ist leider nicht in der Lage, durch vegetative Teilung der einzelnen Möhren (wie bei Zuckerrüben) den Samenertrag einer Einzelpflanze so weit zu steigern, daß ausreichend große Parzellen mit vielfacher Wiederholung angelegt werden können.

Es wäre wünschenswert, entweder eine Methode $\mathrm{zu}$ entwickeln, mit der es gelingt, von der einzelnen Möhrenpflanze mehr Saatgut zu erzeugen, oder, was die gleiche Auswirkung hätte, eine Methode zu entwickeln, die es ermöglicht, mit dem wenigen Saatgut eine größere Fläche so $\mathrm{zu}$ bestellen, daß ein geschlossener Pflanzenbestand erzeugt wird (Saatgutverdünnung usw.).

Bezüglich der Anlage der Einzelpflanzennachkommenschaftsprüfungen verweisen wir auf die nachfolgende Beschreibung der Stammprüfung.

In der zweiten und den folgenden Nachkommenschaften der Einzelpflanzen, die entweder als Stämme oder in Gruppen weiterbearbeitet werden, liegt bereits so viel Saatgut vor, daß exakte Prüfungen durchgeführt werden können.

Prïfung auf Frïhzeitigkeit bei Treibmöhren. Die Aussaat und der Anbau müssen unter den natürlichen Anbaubedingungen von Frühsorten vor sich gehen. Es ist mindestens eine fünfmalige Wiederholung der zu vergleichenden Sorten vorzunehmen. Bei Prüfung im Kasten müssen je Wiederholung mindestens 2 qm große Parzellen Verwendung finden. Nach dem Aufgang müssen die Möhren gleichmäßig in der Reihe auf $2 \mathrm{~cm}$ vereinzelt werden. Bei der Ernte sind Randwirkungen, verursacht durch die Kastenwand oder durch Querhölzer, auszuscheiden. Die Ernte erfolgt am zweckmäßigsten in Stufen, so daß man einen kurvenmäßigen Überblick über den Ertragsverlauf der einzelnen Sorten erhält.

Bei frühen Freilandmöhren ist in gleicher Weise vorzugehen, nur mit dem Unterschied, daß die Parzellen größer gemacht werden. Am zweckmäBigsten sind vierreihige Parzellen von $5 \mathrm{~m}$ Länge, die Wiederholungszahl ist ebenfalls mindestens 5, der Abstand in der Reihe ebenfalls $2 \mathrm{~cm}$. Da die Aussaat der Frühmöhren im Freiland von März bis Juni durchgeführt wird, muß mit entsprechenden Stufenaussaaten gearbeitet werden. 
Zur Feststellung der Frühzeitigkeit ist eine mehrmalige Stufenernte vorzunehmen. Bei Stufenernte müssen Parzellen von mindestens $5 \mathrm{qm}$ geerntet werden.

Bei den Spätmöhren sind die Versuche in entsprechender Weise anzulegen. Man würde mit einer Aussaatzeit auskommen; es ist bei ihnen auch nicht unbedingt erforderlich, eine stufenweise Ernte vorzunehmen. Abstand größer, etwa $5 \mathrm{~cm}$, da die Möhren dick werden.

Um die Schoßneigung zu prüfen, empfiehlt sich für die Sorten der verschiedensten Zuchtrichtungen eine möglichst frühe Freilandsaat.

Um eine Auslese aufNichtplatzen vorzunehmen, ist ebenfạlls eine frühe Aussaat notwendig, und zwar nach Möglichkeit auf Böden, die im Herbst viel Feuchtigkeit aufweisen.

Die Auslese auf Lagerfähigkeit kann man durch einfache normale Mietenlagerung durchführen. Man kann aber auch die Lagerungsbedingungen verschärfen, indem man die Möhren bei höheren Temperaturen, als sie bei der normalen Lagerung vorkommen, einlagert.

2. Methoden zur Erfassung der Eigenschaften, die besonders den Verwerter interessieren. Alle Eigenschaften, die sich auf die äußere Form und Farbe der Möhren beziehen, wie spitze Kopfform, zylindrische Gestalt, glatte Oberfläche, kleine flache Wurzelansätze, wenig Seitenwurzeln, Nichtherauswachsen aus dem Boden, Nichtgrünköpfigkeit, lassen sich mit dem Auge erkennen. Diese Eigenschaften sind aber außerordentlich modifizierbar durch die Wachstumsbedingungen. Es ist notwendig, jeweils die Wachstumsbedingungen $\mathrm{zu}$ wählen, die die Ausprägung der betreffenden Eigenschaft erschweren. Es ist bekannt, daß die Form der Möhren durch schweren und trockenen Boden ungünstig beeinflußt wird. Man wird daher, wenn man besonders formechte Möhren auslesen will, die Auslese auf solchen Böden vornehmen.

Um eine Einzelpflanzenauslese auf die innere Farbe, Form, Geschmacks- und Werteigenschaften vornehmen zu können, muß man die Möhre teilen. In der Zuckerrübenzüchtung bohrt man die Rüben und untersucht den so gewonnenen Brei. Diese Methode ist bei den Möhren nicht anwendbar. Man ist daher gezwungen, die Möhren der Länge nach oder quer zu schneiden. Aus der Schnittfläche kann man die inneren sichtbaren Eigenschaften, wie Farbe und Form des Holz- und Rindenteils, die Struktur u.a., erkennen. Um die Veränderungen, die beim Konservieren in der Farbe des Holzteils, Kambiums und Rindenteils vor sich gehen, feststellen zu können, muß man Ausschnitte der Möhre kochen. Wir konnten feststellen, daß ein rein roter Holzteil und eine rein rote kambiale Zone nach dem Kochen gelb werden können. Gewünscht werden aber Möhren, deren Holzteil auch beim Kochen rot bleibt.

Im abgeschnittenen Teil lassen sich die Untersuchungen auf Geschmacks-, Zucker- und Aromastoffe und auf die Wirkstoffe - insbesondere Karotin - durchführen.

Voraussetzung für eine erfolgreiche Möhrenzüchtung ist daher zunächst eine Methode, bei der man die Rübe so teilt, daß aus dem Rest Saatgut erzeugt werden kann. Es wird Aufgabe der Forschung sein, entsprechende Untersuchungen durchzuführen. Die Hauptschwierigkeit bei der Möhrenprobenahme ist die, daß die Möhre viel leichter nach einer Verletzung verfault als die Zuckerrübe. Man wird also die Schnittflächen entsprechend behandeln müssen.

Wenn man eine Auslese auf Lagerbestänaigkeit der Farbe, Geschmack und seine Teileigenschaften sowie auf Karotingehalt vornehmen will, so muß man die entsprechenden Untersuchungen im Frühjahr durchführen. Hat man Nachkommenschaften $\mathrm{zu}$ beurteilen, liegen die Dinge wesentlich einfacher, weil man bei den Nachkommenschaftsprüfungen keine Teilung der Möhren vorzunehmen braucht, sondern entsprechende Mengen eines jeden Stammes zur Untersuchung heranziehen kann.

3. Methoden zur Erfassung der Eigenschaften, die den Verbraucher interessieren. Die Zuckeruntersuchung der Möhre liegt wesentlich komplizierter als bei der Zuckerrübe. Bei der Zuckerrübe haben wir fast ausschließlich mit dem Disaccharid (Rohrzucker) zu tun, während in der Möhre neben Disacchariden auch Monosaccharide vorkommen. Man muB daher, um den Süßwert der Möhre festzustellen, die einzelnen Zuckerarten nebeneinander bestimmen. Aus diesem Grunde hat auch die Anwendung der Refraktometermethode bei Möhren nicht ohne weiteres die Aussichten wie bei der Zuckerrübe.

Wenn man möglichst süß schmeckende Möhren erhalten will, könnte man daran denken, den Rohrzuckergehalt der Möhre besonders stark zu erhöhen, weil dieser unter den Zuckerarten der Möhre den höchsten Süßwert hat. Züchterisch brauchbare Schnellbestimmungsmethoden zur Erfassung der einzelnen Zuckerarten nebeneinander liegen nicht vor.

Bereits vor Jahren hat BECKER-DILLINGEN die Refraktometermethode für die Möhrenuntersuchung empfohlen. Trotz der theoretisch vorhandenen Schwierigkeiten wurde versucht, die 
Refraktometermethode zur Bestimmung des Zuckergehalts zu verwenden. Der abgepreßte Möhrensaft läßt sich nicht refraktometern, weil die Lösung trübe und dadurch keine einwandfreie Ablesung möglich ist. Aus diesem Grunde wurde von uns ein Verfahren entwickelt, das diesem Übelstand abhilft. Es wird eine bestimmte Gewichtsmenge roh zerkleinerter Möhren mit der gleichen Menge Wasser versetzt und in einem Reagenzglas mit Watteverschluß im Dampftopf $\mathrm{I}^{1} / 2$ Std. bei $100^{\circ} \mathrm{C}$ erhitzt. Die Lösung läßt sich jetzt ohne weiteres refraktometern, d. h. ermöglicht eine eindeutige Ablesung der Werte. Die Refraktometerwerte liegen natürlich entsprechend niedriger als bei Rohsaft.

Bei der Untersuchung von vielen Einzelmöhren ergeben sich wesentliche Unterschiede im Refraktometerwert. Prüft man das Restmaterial derjenigen Rüben mit hohem Refraktometerwert, so findet man, daß diese in der Regel süßer schmecken als die mit niedrigem Refraktometerwert.

Wenn also auch die Schwierigkeiten bestehen, die sich aus der Anwesenheit von Mono- und Disacchariden in der Möhre ergeben, so läßt sich vielleicht doch die Refraktometermethode zur rohen Vorauslese verwenden.

Zur Bestimmung des Karotingehalts liegen heute noch keine züchterisch brauchbaren chemischen und biologischen Schnellbestimmungsmethoden vor. Es wäre erwünscht, wenn die Forschung entsprechende Schnellbestimmungsmethoden entwickeln würde.

Auch zur Prüfung der Konsistenz haben wir heute noch keine geeigneten Methoden. Auch hier müßte man versuchen, entsprechende Apparaturen für die Prüfung zu entwickeln.

Zusammenfassend können wir feststellen, da $\beta$ die Methoden der Möhrenauslese und -prüfung noch in keiner Weise den praktischen Anforderungen genügen und daß zunächst sehr intensiv an der Entwicklung geeigneter Methoden gearbeitet werden $\mathrm{muB}$.

\section{Pflanzenmaterial.}

Wenn man heute das Sortiment der Möhren prüft, so findet man, daß sortenmäßige Unterschiede bestehen (SchuphaN), daß aber die Sorten selbst in sich jeweils nicht einheitlich sind, sondern ein mehr oder weniger großes Typengemisch darstellen.

Wir haben innerhalb verschiedener Sorten die Variationsbreite der einzelnen Eigenschaften festgestellt. Es konnten bezüglich der oben erwähnten Eigenschaften außer- ordentlich extreme Formen gefunden werden. So waren in den meisten Sorten Pflanzen vertreten, die die oben erwähnten Eigenschaften in der positiven und negativen Form zeigten. Besonders hervorgehoben seien hier die Pflanzen mit besonders dunkelroter Farbe, mit besonders gutem Geschmack und mit besonderer Haltbarkeit der tiefdunklen Farbe nach dem Kochen und nach längerer Lagerzeit. Die meisten der genannten Eigenschaften konnten in ihrer positiven Ausprägung in den untersuchten Sorten gefunden werden, d.h. da $B$ unsere heutigen Sorten bereits ein günstiges Ausgangsmaterial für die züchterische Auslese darstellen. Leider sind aber die Pflanzen, die eine Kombination vieler gewünschter Eigenschaften aufweisen, außerordentlich selten. Wir werden daher sehr schnell ein Sortiment von Typen auffinden können, das jeweils eine der genannten Eigenschaften in idealer Form aufweist. Wenn wir mehrere der genannten Eigenschaften in idealer Form auf einer Pflanze vereinigt finden wollen, müssen wir ein sehr großes Einzelpflanzenmaterial der Auslese unterziehen. Es besteht aber kein Zweifel darüber, daß es gelingen wird, eine Reihe der gewünschten Eigenschaften bereits heute kombiniert vorzufinden.

$$
\text { IV. Auslese. }
$$

Die Auslese selbst wird man zunächst an Einzelpflanzen der verschiedenen besonders geeigneten Sorten vornehmen. Wir haben gesehen, daß es notwendig ist, eine sehr große Zahl von Pflanzen zu untersuchen, um die gewünschten idealen Ausprägungen der Eigenschaften $\mathrm{zu}$ finden. Von SENGBusch hat in diesem Zusammenhang den Vorschlag gemacht, die Auslese „vor oder hinter der Waschmaschine" der Konservenindustrie vorzunehmen. Diese Methode hätte den Vorteil, daß man ein fast unendlich großes Ausgangsmaterial zur Verfügung hat, das man nicht nur auf seine äußere Form, sondern nach einem entsprechenden Teilen auch auf seine inneren Eigenschaften untersuchen kann.

Die Auslese in der Konservenfabrik hätte außerdem den großen Vorteil, daß der Züchter in dauernder Fühlung mit dem Leiter und dem Techniker der Konservenfabrik steht und sich über die Bedeutung der einzelnen Eigenschaften für die Konservierung ein klares Bild machen kann. Es dürfte nach der ersten Auslese der idealen Ausprägung der Einzeleigenschaften und der anschließenden Stammprüfungen möglich sein, die Stämme in sehr viel größerem Umfang zu prüfen, als das in einem normalen Zuchtbetrieb möglich wäre. 
Wir haben schon mehrfach darauf hingewiesen, daß es bei züchterischen Arbeiten nicht gleichgültig ist, in welcher Reihenfolge die Bearbeitung der einzelnen Zuchtziele vorgenommen wird. Es sei daher auch in dieser Zusammenstellung kurz auf die Bedeutung der einzelnen Möhreneigenschaften hingewiesen. Zunächst wird man innerhalb der einzelnen Gruppen auf gute Form und tiefrote Farbe (innerhalb von rooooo und mehr Individuen) auslesen. Erst wenn diese Zuchtziele in Kombination mit den Gruppeneigenschaften (Frühreife oder Ertrag) verwirklicht sind, wird man die Geschmacksprüfung vornehmen. Es scheint zweckmäßig zu sein, bei der Geschmacksprüfung eine Vorauslese mit Hilfe des Refraktometers (an vielen Tausend Möhren) durchzuführen. Innerhalb der Möhren mit hohem Refraktometerwert (einige Hundert Individuen) werden Geschmacksproben vorgenommen. Anschließend kann man dann die Karotinprüfung durchführen, und zwar aus dem Grunde, weil schlecht schmeckende Möhren, auch wenn sie viel Karotin enthalten, keine Verwendung finden können; ferner weil man die Geschmacksprüfung leichter in einer großen Zahl durchführen kann als vorläufig die Karotinbestimmung.

Die Auslese auf Farbe würden wir ebenfalls der Karotinprüfung voranstellen, weil $\mathrm{u}$. E. die Wahrscheinlichkeit, unter tiefdunkelrot gefärbten Möhren karotinreiche Formen $\mathrm{zu}$ finden, größer ist als unter blaßroten. Farbe und Karotingehalt ist jedoch nicht das gleiche, und daher werden unter den dunkelrot gefärbten Möhren auch karotinarme Formen vorhanden sein. Die Wahrscheinlichkeit ist aber gro $B, \mathrm{da} B$ man unter den farbstoffreichsten auch die karotinreichsten Möhren finden wird.

In diesem Zusammenhang sei auch auf die außerordentlich interessante und aufschlußreiche Arbeit von SchupHaN hingewiesen.

Nach der Auslese der bezüglich der einzelnen Eigenschaften idealen Pflanzen wird man diese untereinander kreuzen. In der Nachkommenschaft dieser Kreuzungen wird man nach Pflanzen suchen, die eine möglichst große Zahl der genannten Eigenschaften in idealer Ausprägung aufweisen.

Mit der Auslese selbst ist es aber bei dem Fremdbefruchter Möhre nicht getan. Wir müssen vielmehr die jeweilig ausgelesenen positiven Einzeleigenschaften für die Kombination der Einzeleigenschaften erhalten. Wir können so vorgehen, daß wir Eigenschaftsgruppen zusammenstellen und die Befruchtung jeweils innerhalb einer Gruppe (Massenkreuzung) vor sich gehen lassen. Wir können aber auch inner- halb jeder Gruppe Paarkreuzungen vornehmen, um u. U. leichter Pflanzen ausmerzen zu können, die die ideale Ausprägung der einzelnen Eigenschaften nur phänotypisch aufweisen.

Bei dem heutigen Stand der Möhrenzüchtung dürfte es wahrscheinlich praktisch sein, aus einer sehr großen Zahl von Einzelpflanzen die idealen Formen herauszufinden, sie in Gruppen zusammenzustellen und Gruppenkreuzungen zu machen. Erst später mit weiterem Fortschreiten der züchterischen Arbeiten wird man die Paarkreuzungen zum $Z$ wecke der Stabilisierung der Einzeleigenschaften anwenden.

Nach der Fixierung der gewünschten Eigenschaften und evtl. Kombination von einer Reihe von Eigenschaften wird man die Neuzüchtungen, soweit sie den vorhandenen Sorten bereits überlegen sind, als Neuzüchtungen anmelden und ihre Zulassung abwarten.

\section{Erhaltungszüchtung.}

Ganz besonders nachdrücklich soll darauf hingewiesen werden, daß die züchterische Arbeit an Fremdbefruchtern nicht mit der erfolgten $\mathrm{Zu}$ lassung aufhört, sondern daß an den Sorten eine intensive Erhaltungszüchtung betrieben werden muB. Diese Erhaltungszüchtung erschöpft sich aber nicht in der Erhaltung der ursprünglichen Leistungseigenschaften, sondern sie ist die Grundlage für eine weitere Steigerung aller Werteigenschaften.

Bei unseren landwirtschaftlichen Kulturarten ist diese Erhaltungszüchtung bei Fremdbefruchtern eine Selbstverständlichkeit geworden, und es ist zu wünschen, daß auch in der Gemüsezüchtung die Erhaltungszüchtung mit der gleichen Intensität durchgeführt wird.

\section{Saatguterzeugung.}

Die Art der Verbrauchssaatguterzeugung spielt für die Erhaltung der Werteigenschaften einer Neuzüchtung eine erhebliche Rolle.

Bisher wurden die Möhren in Deutschland züchterisch bearbeitet und die Saatguterzengung zum großen Teil in klimatisch besonders begünstigten Gebieten Frankreichs durchgeführt. Die zu vermehrenden Sorten wurden dort ausgedrillt, überwintert und lieferten Saatgut. Diese Art der Vermehrung brachte uns in eine wirtschaftliche Abhängigkeit bezüglich der Möhrensaatguterzeugung vom Ausland. Außerdem hat die Sämlingssaatguterzeugung bei Möhren ebenso wie bei den Kohlarten den Nachteil, daB die Werteigenschaften der Sorten zurückgehen, die negativen Typen sich unverhältnismäßig stark vermehren und der Sortencharakter ungünstig 
beeinflußt wird. Wir müssen daher vor allen Dingen bei Möhrenneuzüchtungen genau wie bei Kohl die Saatguterzeugung aus voll ausgewachsenen Pflānzen vornehmen. Diese ausgewachsenen Pflanzen werden vor dem Auspflanzen einer Auslese unterworfen. Erst wenn der Sortencharakter weitgehend gefestigt ist, wird man evtl. zu einer einmaligen Sämlingsvermehrung bei der Erzeugung von Hochzuchtsaatgut übergehen können. Es wäre wünschenswert, wenn die Vermehrung von Möhrensaatgut zu einem großen Teil in Deutschland selbst vorgenommen wird, so daß eine Abhängigkeit vom Ausland nicht eintreten kann. Ferner müßte man bei guten Möhrensorten dazu kommen, daß man wie früher bei Zuckerrüben etwa 50 bis $80 \%$ des Saatgutbedarfs überlagert, so daß Mißernten die Saatgutversorgung nicht gefährden können.

Voraussetzung für eine derartige Vermehrung von Möhrensaatgut aus voll ausgewachsenen Pflanzen ist, daß die Vermehrerpreise so gestaltet werden, daß der Vermehrer eine Saatguterzeugung aus voll ausgewachsenen Pflanzen durchführen kann.

\section{Schluß.}

Wir haben versucht, in der vorliegenden kurzen Zusammenstellung eine Übersicht über die wichtigsten Zuchtziele, die der Anbauer, der Verwerter und der Verbraucher stellen, zu geben und anschließend die Möglichkeiten der Erreichung dieser Zuchtziele erörtert. Es sei hier zum $\mathrm{Ab}$ schluß noch einmal auf den Gedanken der Auslese in Verbindung mit der Konservenindustrie und in der Konservenindustrie selbst hingewiesen. Wir möchten ferner der Hoffnung Ausdruck geben, daß es gelingen möge, die Saatguterzeugung bei Möhren durch einen entsprechenden Anbau im Inland so in die Hand zu bekommen, $\mathrm{daB}$ sich Schwierigkeiten in der Saatgutversorgung nicht einstellen können. Die wirtschaftlichen Voraussetzungen für den Inlandanbau werden sich schaffen lassen.

Forschungsaufgaben.

I. Vergleichende Prüfung der Ausleseverfahren.

a) Massenkreuzungen, b) Paarkreuzungen,

c) Bedeutung der Inzucht.

2. Methode zum Teilen der Möhre, wobei ein Teil zur Gewinnung von Saatgut Verwendung findet und der andere zur Durchführung der Qualitätsuntersuchung dient.

3. Die Ursache für die Haltbarkeit bzw. Nichthaltbarkeit der roten Färbung der Möhren.

4. Die Ursache des Umschlags der roten Färbung des Holzteils und der kambialen Zone von rot in gelb durch das Kochen.

5. Vergleichende Untersuchungen über die Beziehungen zwischen dem Geschmack auf der einen Seite und dem Refraktometerwert und dem Gehalt an verschiedenen Zuckerarten auf der anderen Seite.

6. Studium der positiven und negativen Geschmacksstoffe und evtl. Ausarbeitung geeigneter. Methoden zur chemischen Bestimmung dieser Geschmacksstoffe.

7. Ausarbeitung von chemischen Schnellmethoden für die Karotinbestimmung.

8. Ausarbeitung von biologischen Schnellmethoden für die Karotinbestimmung.

9. Studium der Beziehungen zwischen chemisch nachweisbarem Karotin und dem biologisch aufnehmbaren Karotin.

ro. Entwicklung von geeigneten Verfahren, mit wenig Saatgut eine exakte Leistungsprüfung von Einzelmöhrennachkommenschaften durchzuführen.

II. Die Durchführung der Leistungsprüfungen von Möhrenstämmen bzw. Gruppennachkommenschaften.

I2. Entwicklung eines Apparates zur Bestimmung der Konsistenz von Möhren.

I3. Prüfung der Frage, welche Böden für die Auslese besonders formechter und farbechter Möhren vorwiegend geeignet sind.

\section{Literatur.}

Becker-Dilimangen, J.: Handbuch des Gemüsebaues, 2, Aufl. Verlag Paul Parey, Berlin. Kreula, M., u. A. I. Virtanen: Z. physiol. Chem. 270, I4I-I52 (I94I). - NicolaiseN, NrColaI: Handb. Pflanzenzüchtg I939, V. Bd. - ScH UPHAN, W.: Züchter I942, Heft 2. - ViRTanen, A. I.: Forsch.dienst I3, Heft I (1942). - Virtanen, A. I.: Scientia (Milano) 35, 28-37 (I94I). - VirTaneN, A. I., S. v. Hausen u. S. Saastamoinen: Biochem. Z. 267 , I79-I9I (I933). 